University of Nebraska - Lincoln

DigitalCommons@University of Nebraska - Lincoln

$1-1-2006$

\title{
Bioelectromechanical imaging by scanning probe microscopy: Galvani's experiment at the nanoscale
}

Sergei V. Kalinin

Condensed Matter Sciences Division, Oak Ridge National Laboratory, Oak Ridge, TN, sergei2@ornl.gov

B. J. Rodriguez

Condensed Matter Sciences Division, Oak Ridge National Laboratory, brian.rodriguez@ucd.ie

J. Shin

Condensed Matter Sciences Division, Oak Ridge National Laboratory

Stephen Jesse

Oak Ridge National Laboratory, sjesse@ornl.gov

V. Grichko

North Carolina State University, Raleigh

See next page for additional authors

Follow this and additional works at: https://digitalcommons.unl.edu/physicsgruverman

Part of the Physics Commons

Kalinin, Sergei V.; Rodriguez, B. J.; Shin, J.; Jesse, Stephen; Grichko, V.; Thundat, T.; Baddorf, Arthur P.; and Gruverman, Alexei, "Bioelectromechanical imaging by scanning probe microscopy: Galvani's experiment at the nanoscale" (2006). Alexei Gruverman Publications. 41.

https://digitalcommons.unl.edu/physicsgruverman/41

This Article is brought to you for free and open access by the Research Papers in Physics and Astronomy at DigitalCommons@University of Nebraska - Lincoln. It has been accepted for inclusion in Alexei Gruverman Publications by an authorized administrator of DigitalCommons@University of Nebraska - Lincoln. 


\section{Authors}

Sergei V. Kalinin, B. J. Rodriguez, J. Shin, Stephen Jesse, V. Grichko, T. Thundat, Arthur P. Baddorf, and Alexei Gruverman 


\title{
Bioelectromechanical imaging by scanning probe microscopy: Galvani's experiment at the nanoscale
}

\author{
Sergei V. Kalinin ${ }^{\mathrm{a}, *}$, B.J. Rodriguez ${ }^{\mathrm{a}, \mathrm{b}}$, J. Shin ${ }^{\mathrm{a}, \mathrm{c}}$, S. Jesse $^{\mathrm{a}}$, V. Grichko ${ }^{\mathrm{d}}$, T. Thundat ${ }^{\mathrm{e}}$, \\ A.P. Baddorf ${ }^{\text {a }}$ A. Gruverman ${ }^{f}$ \\ ${ }^{a}$ Condensed Matter Sciences Division, Oak Ridge National Laboratory, Oak Ridge, TN 37831, USA \\ ${ }^{\mathrm{b}}$ Department of Physics, North Carolina State University, Raleigh, NC 27695, USA \\ ${ }^{\mathrm{c}}$ Department of Physics and Astronomy, University of Tennessee, Knoxville, TN 37996, USA \\ ${ }^{\mathrm{d}}$ Department of Biochemistry, North Carolina State University, Raleigh, NC 27695, USA \\ ${ }^{\mathrm{e}}$ Life Sciences Division, Oak Ridge National Laboratory, Oak Ridge, TN 37831, USA \\ ${ }^{\mathrm{f}}$ Department of Materials Science and Engineering, North Carolina State University, Raleigh, NC 27695, USA
}

Received 15 June 2005; received in revised form 25 October 2005; accepted 26 October 2005

\begin{abstract}
Since the discovery in the late 18 th century of electrically induced mechanical response in muscle tissue, coupling between electrical and mechanical phenomena has been shown to be a near-universal feature of biological systems. Here, we employ scanning probe microscopy (SPM) to measure the sub-Angstrom mechanical response of a biological system induced by an electric bias applied to a conductive SPM tip. Visualization of the spiral shape and orientation of protein fibrils with $5 \mathrm{~nm}$ spatial resolution in a human tooth and chitin molecular bundle orientation in a butterfly wing is demonstrated. In particular, the applicability of SPM-based techniques for the determination of molecular orientation is discussed.
\end{abstract}

(C) 2005 Elsevier B.V. All rights reserved.

PACS: 77.84.--s; 87.64.Dz; 82.37.Gk

Keywords: Scanning probe microscopy; Piezoresponse force microscopy; Piezoelectricity; Nanoscale; Proteins

\section{Introduction}

Performed more than 200 years ago, experiments on muscular contraction in a frog under an electric bias [1] were the first observation of the electromechanical coupling effect in biological systems. One of the most important manifestations of electromechanical behavior is piezoelectricity, which stems from the crystal structure of most biopolymers including cellulose, collagen, keratin, etc. Piezoelectric behavior has been observed in a variety of biological systems including bones [2-5], teeth [6], wood [7,8], and seashells [9]. It has been postulated that piezoelectric coupling, via mechanical stress that generates an

\footnotetext{
${ }^{*}$ Corresponding author.

E-mail addresses: sergei2@ornl.gov (S.V. Kalinin), alexei_gruverman@ncsu.edu (A. Gruverman).
}

electric potential, controls the mechanisms of local tissue development $[10,11]$. Understanding the relationship between physiologically generated electric fields and mechanical properties on the molecular, cellular and tissue levels has become the main motivation of studying piezoelectricity in biological systems.

In inorganic materials, such as quartz, the piezoelectric properties are typically measured on single-crystalline samples, and these properties can be projected to any length scale from micro- to nanoscopic. In contrast, biological materials are composed of dissimilar structural elements arranged in a complex hierarchical structure, each level bringing new aspects to the overall properties of the material. Hardness and fracture strength exhibited on a micrometer level by biological materials, such as bone or wood, are due to the staggered configuration of nanoscale platelets of hard phase (for example, hydroxyapatite 
(HAP) crystals in bones, aragonite in shells, cellulose in wood) embedded in a soft matrix (collagen or lignin) [12]. In bones, the arrangement of mineralized collagen fibrils gives rise to as many as 7 levels of structural organization [13]. Characterization of local molecular orientation and electromechanical and mechanical properties in biological systems at different length scales down to the individual building blocks of biomaterials is the key to unraveling the fundamental mechanisms of tissue growth and regeneration. Previous studies were performed on the averaged level using small angle X-ray scattering [14] or locally by electron microscopy [15], or by destructive nano-indentation techniques [16]. However, information on local properties on the sub-100 $\mathrm{nm}$ level has been sparse [17]. Recently, piezoelectric measurements of biosystems with sub- $10 \mathrm{~nm}$ resolution [18] and combined elasticity and electromechanical measurements of a wide range of biosystems [19,20] has been demonstrated. In this paper, we demonstrate nanoscale imaging of electromechanical behavior in biological systems and describe an approach to determine molecular orientation with sub- $10 \mathrm{~nm}$ resolution using a combination of scanning probe microscopy (SPM) techniques. Piezoelectric properties are measured semiquantitatively within a single $150 \mathrm{~nm}$ protein fibril. Using vector electromechanical measurements, the orientation of protein molecules can potentially be determined in real space, providing a novel structural characterization tool for biological systems.

\section{Materials and methods}

The local electromechanical properties on the nanoscale are accessed by piezoresponse force microscopy (PFM) $[21,22]$. To measure the piezoelectric properties of a sample, PFM is performed in contact mode with the application of a periodic electrical bias, $V_{\text {tip }}=V_{\mathrm{dc}}+$ $V_{\text {ac }} \cos \omega t$, between a conductive SPM tip and the backside of the sample. This bias results in a periodic displacement of the surface, $d=d_{1 \omega} \cos (\omega t+\varphi)$, which can be measured with sub-Angstrom precision (typical piezoelectric displacements for $10 V_{\mathrm{pp}}$ driving voltage are $\sim 30-40 \mathrm{pm}$, corresponding to coupling coefficients on the order of $\sim 3-4 \mathrm{pm} / \mathrm{V})$. The interaction volume beneath the tip (i.e. the volume that is piezoelectrically excited) depends on the contact radius, the applied bias and local properties of the material, and is generally of the order of 5-20 nm, providing a measure of spatial resolution and field penetration in the material. The amplitude and phase of the cantilever oscillations reveal information on the strength and sign of the local electromechanical response, respectively. Both vertical and lateral components of surface displacement can be detected. Vertical PFM (VPFM) measurements have been performed at frequencies of $50-100 \mathrm{kHz}$, which minimizes the longitudinal contribution to measured vertical signal [23]. For lateral PFM (LPFM), the optimal conditions for contrast transfer were $\sim 10 \mathrm{kHz}$; for higher frequencies, the onset of sliding friction minimizes in-plane oscillation transfer between the tip and the surface [23,24].

Complementary information on local mechanical properties is obtained using atomic force acoustic microscopy (AFAM) [25]. For AFAM measurements, the samples are glued to a commercial lead zirconium titanate (PZT) oscillator [26]. The sample is then vibrated mechanically by the piezoelectric actuator and acoustical waves transmitted to the tip are detected, providing a contrast between hard and soft regions of the sample.

PFM and AFAM are implemented on a commercial SPM (Veeco MultiMode NS-IIIA) equipped with additional function generators and lock-in amplifiers (DS 345 and SRS 830, Stanford Research Instruments, and Model 7280, Signal Recovery) as described elsewhere [19]. A custom-built sample holder was used to allow direct tip biasing and to avoid capacitive cross-talk in the SPM electronics. Measurements were performed using Pt- and Au-coated tips (NSC-12 C, Micromasch, $l=130-\mu \mathrm{m}$, resonant frequency $\sim 150 \mathrm{kHz}$, spring constant $k \sim 4.5 \mathrm{~N} /$ $\mathrm{m})$. In general, $10 V_{\mathrm{pp}}$ was applied to the tip for PFM measurements, while $1 V_{\mathrm{pp}}, 1 \mathrm{MHz}$ was applied to the bottom electrode of the actuator for elasticity (AFAM) measurements. The typical scan rate was $1 \mathrm{~Hz}$. AFAM, vertical and lateral PFM signals were collected simultaneously with perfect spatial correlation (Fig. 1) using custom LabView software [19]. Grounding the top electrode of the oscillator was sufficient to minimize cross-talk between PFM and AFAM signals. PFM provides a unique tool to measure piezoelectric properties on the nanoscale since piezoelectric response is tied to crystal structure, and hence to the orientation of individual molecules in biological materials. As a result, probing electromechanical response by PFM is a measure of molecular orientation for piezoelectric molecules.

\section{Results and discussion}

\subsection{Tooth sample}

A PFM-AFAM approach has been used to perform mechanical and electromechanical imaging in a human tooth. The deciduous human tooth sample was crosssectioned parallel to the growth direction and polished to a thickness of $\sim 0.5 \mathrm{~mm}$ using diamond polishing pads down to $0.5 \mu \mathrm{m}$ grit size. The sample was subsequently mounted on the oscillator using silver paint. The enamel and dentin regions can be readily identified using optical microscopy. PFM images of dissimilar dental tissues are illustrated in Fig. 2. A strong response signal of the dentin region is consistent with a high density of piezoelectrically active collagen [6]. Several isolated regions with a high piezoresponse signal are observed in the enamel region. Since enamel is composed primarily of centrosymmetric, hence, non-piezoelectric HAP, we attribute the piezoresponse signal to a low fraction of protein fibers present in the enamel. 


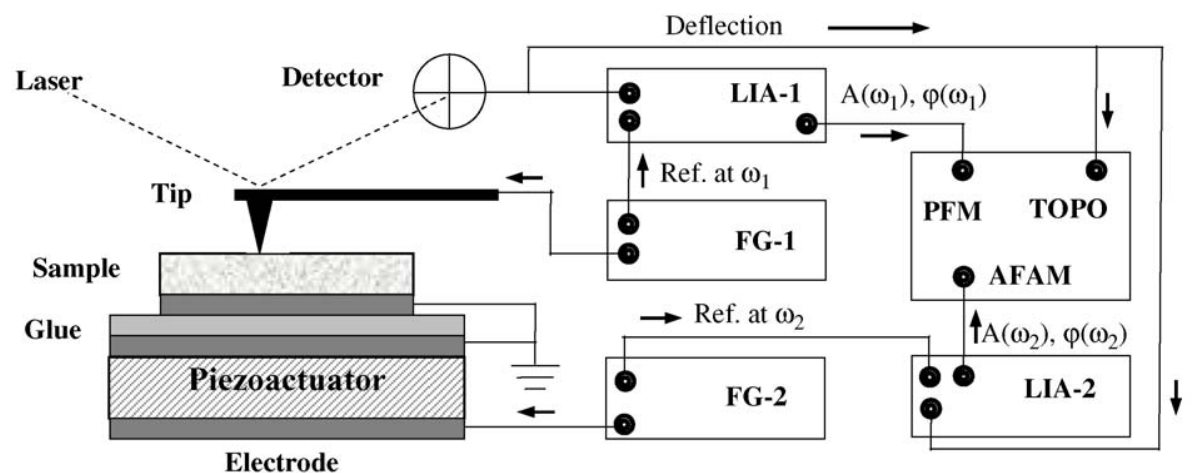

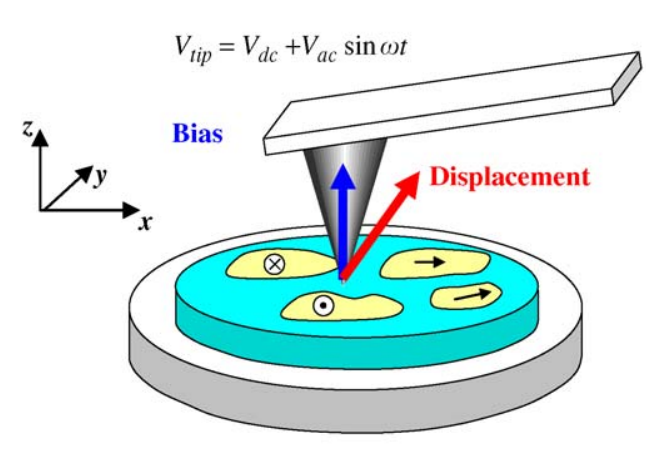

(a)

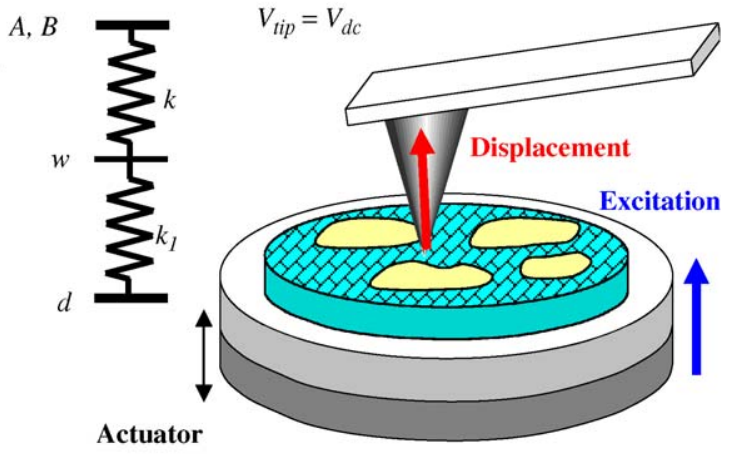

(b)

$$
d=d_{0}+\delta d \sin \omega_{2} t
$$

Fig. 1. Experimental set-up for PFM/AFAM measurements. Function generator FG-1 biases the tip and the bias-induced tip deflection measured by lockin amplifier LIA-1 constitutes the PFM signal. Function generator FG-2 biases the piezoactuator and the vibration-induced tip deflection measured by lock-in amplifier LIA-2 constitutes the AFAM signal. (b) Schematics of signal formation mechanism, in (b) PFM and (c) AFAM. (Color figures can be viewed in web.)

To get further insight into the structure of the protein inclusion, we have combined measurements of the vertical (out-of-plane) electromechanical response with lateral (inplane) response measurements (Fig. 3a) [21]. Neither topographic (Fig. 3b) nor elastic (Fig. 3c) images of the enamel surface show any significant contrast difference between the topographic features. In comparison, both vertical PFM (VPFM) and lateral PFM (LPFM) images show a strong (signal to noise ratio $>4: 1$ ) electromechanical response that we attribute to a protein fibril embedded within a non-piezoelectric matrix (Fig. 3d, e). The spatial resolution of PFM, determined as the half-width of the boundary between piezoelectric regions with different orientation, is about $5 \mathrm{~nm}$. Note that the resolution achieved is an order of magnitude better than $50-100 \mathrm{~nm}$ typical for single crystals and is comparable to the best results achieved to date for thin films of ferroelectric perovskites. In PFM, in the strong indentation regime [22], the resolution is limited by the tip-surface contact area. For a tip-surface indentation force on the order of $\sim 100 \mathrm{nN}$, the contact radius can be estimated as $\sim 5-20 \mathrm{~nm}$ depending on the tip radius of curvature and the effective Young's modulus of the material. Here, this limit is achieved for a biological material. Given the strong orientation dependence of the PFM signal, this opens a pathway to molecular orientation imaging at comparable resolution.
Comparison of the VPFM and LPFM images reveals a different pattern of piezoelectric domains, suggesting a complicated fibril structure, most likely consisting of several protein molecules. To visualize the electromechanical response data, we employ a vector representation for PFM [23]. The VPFM and LPFM images are normalized with respect to the maximum and minimum values of the signal amplitude so that the intensity changes between -1 and 1 , i.e. $v p r, 1 p r \in(-1,1)$. Using commercial software [27], this 2D vector data (vpr, lpr) is converted to the amplitude/angle pair, $A_{2 \mathrm{D}}=\mathrm{Abs}(\mathrm{vpr}+I \mathrm{lpr}), \theta_{2 \mathrm{D}}=\mathrm{Arg}$ $(\mathrm{vpr}+I \mathrm{lpr})$. These data are plotted so that the color corresponds to the orientation, while color intensity corresponds to the magnitude, as shown in color wheel diagram. The color-encoded vector response map (Vector PFM), shown in Fig. 3f, clearly delineates a helical structure, visualizing the electromechanically active protein fibril conformation in real space [28]. Note the additional details (complex spiral shape of the molecule) that can be visualized in the $2 \mathrm{D}$ vector PFM map, as compared with original scalar data sets.

\subsection{Molecular orientation from PFM data}

This analysis can be extended to create a semiquantitative nanoscale map of local molecular orientation. The electromechanical properties of solids are characterized by 

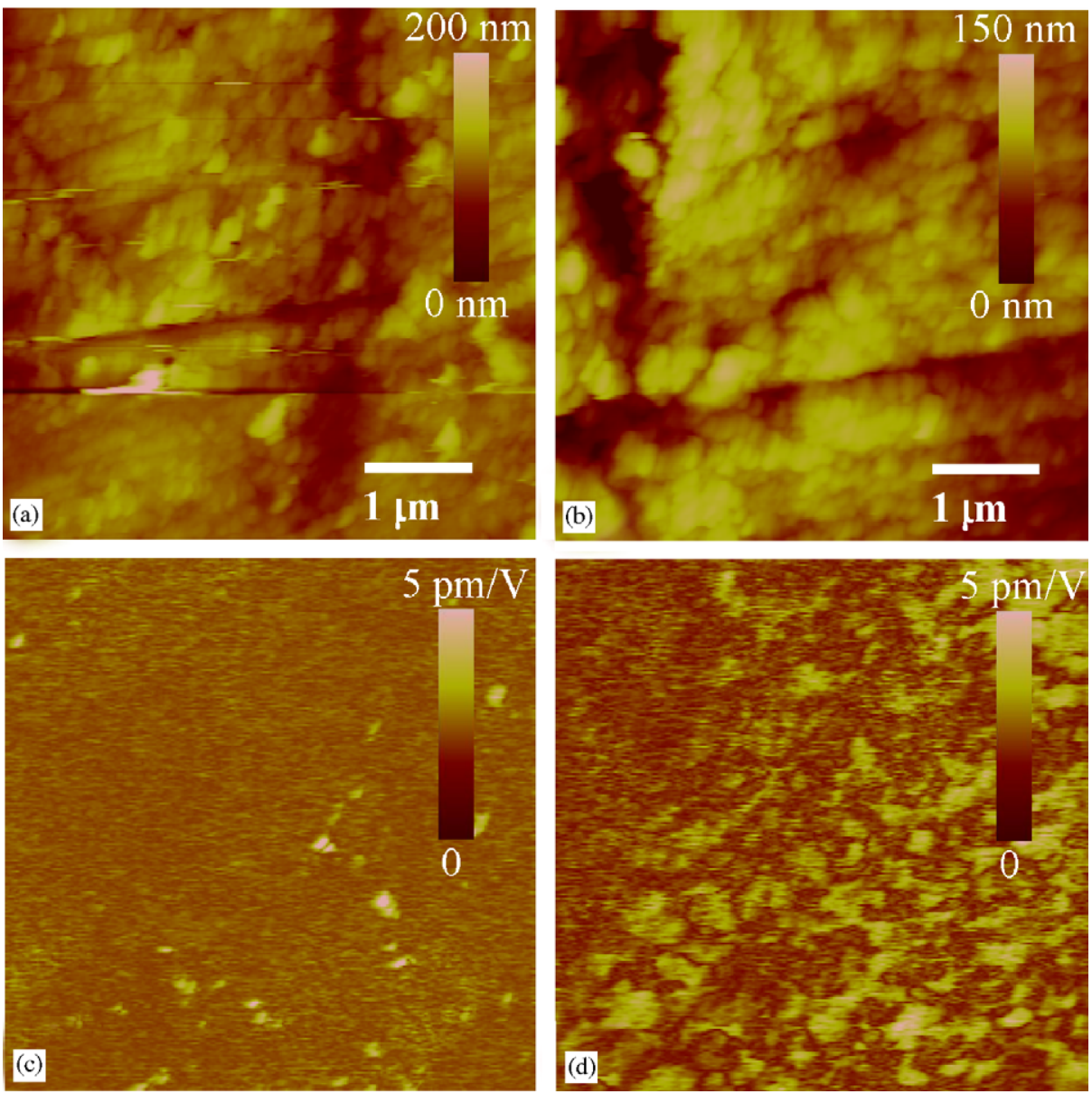

Fig. 2. Topographic (a, c), and piezoelectric (b, d) images of enamel (a, b) and dentin (c, d) regions of dry tooth.

a piezoelectric tensor, $d_{i j}^{0}$, where tensor elements are determined in the coordinate system linked to the principal crystallographic axes. The experimentally measured VPFM and LPFM signals are determined by the coefficients $d_{33}$ and $d_{34}$ of the piezoelectric tensor, $d_{i j}$, in the laboratory coordinate system [23]. The two coordinate systems are related by three Euler rotation angles, $(\theta, \psi, \varphi)[29]$, that uniquely define the local crystallographic orientation in the laboratory coordinate system. The relationship between the $d_{i j}$ tensor in the laboratory coordinate system and the $d_{i j}^{0}$ tensor in the crystal coordinate system is [29]

$d_{i j}=A_{i k} d_{k l}^{0} N_{l j}$,

where $N_{i j}$ and $A_{i j}$ are the rotation matrices. For materials with known $d_{i j}^{0}$ tensor, the local crystallographic orientation, $\left(\phi_{i}, \theta_{i}, \psi_{i}\right)$, can be derived by solving Eq. (1). The detailed theoretical analysis of electromechanical orientational imaging by Vector PFM including approaches to calibration and measurement artifacts is developed elsewhere [23].

For the protein fibril studied in this work, only partial information on the electromechanical response vector is available from VPFM and LPFM data. Assuming that the fibril is formed by collagen (there are few papers that report data on the piezoelectric properties of biomolecules and collagen is chosen as an example), the $d_{i j}^{0}$ matrix has the form [30]

$\left(\begin{array}{cccccc}0 & 0 & 0 & d_{14}^{0} & d_{15}^{0} & 0 \\ 0 & 0 & 0 & d_{15}^{0} & -d_{14}^{0} & 0 \\ d_{31}^{0} & d_{31}^{0} & d_{33}^{0} & 0 & 0 & 0\end{array}\right)$.

From Eq. (1), the components of PFM signal are thus

$$
\begin{aligned}
d_{33}= & 0.5 \cos \theta\left(d_{15}^{0}+d_{31}^{0}+d_{33}^{0}\right. \\
& \left.-\left(d_{15}^{0}+d_{31}^{0}-d_{33}^{0}\right) \cos 2 \theta\right), \\
d_{34}= & \left(-\cos \psi\left(d_{31}^{0}-d_{33}^{0}+\left(d_{15}^{0}+d_{31}^{0}-d_{33}^{0}\right) \cos 2 \theta\right)\right. \\
& \left.-d_{14}^{0} \cos \theta \sin \psi\right) \sin \theta, \\
d_{34}= & \left(-\sin \psi\left(d_{31}^{0}-d_{33}^{0}+\left(d_{15}^{0}+d_{31}^{0}-d_{33}^{0}\right) \cos 2 \theta\right)\right. \\
& \left.+d_{14}^{0} \cos \theta \cos \psi\right) \sin \theta .
\end{aligned}
$$

In this case, the molecule is rotationally invariant and the response is independent on the third Euler angle, $\varphi$. Orientation dependence of PFM signal surfaces are shown in Fig. 4a, b. Note that while exact shape of response surfaces will be strongly dependent on the values of the $d_{i j}^{0}$, 

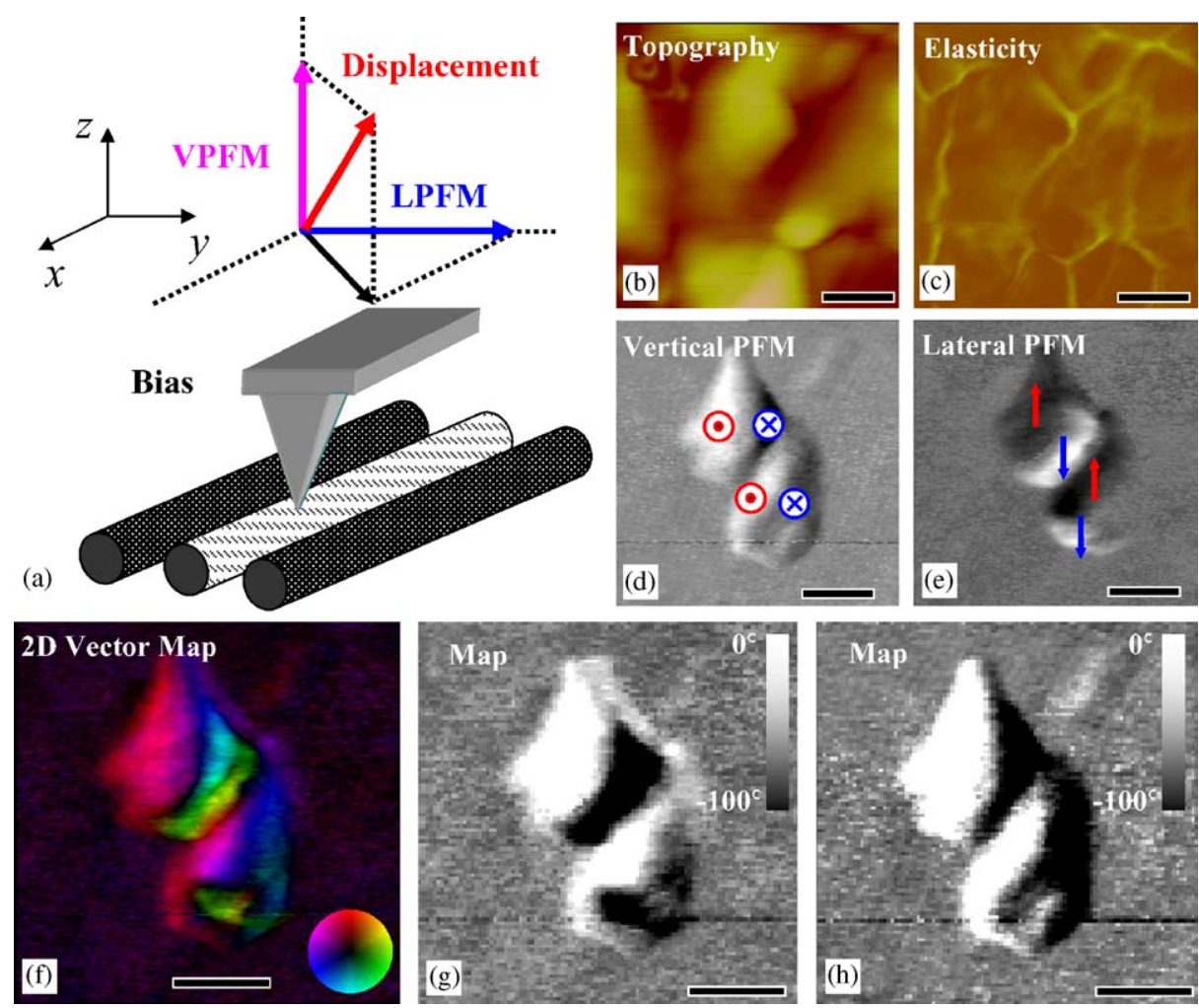

Fig. 3. (a) Electromechanical response of the surface to the tip bias is a vector measure, components of which are related to the local orientation of protein molecules. In PFM, detection of vertical (VPFM) and torsional (LPFM) components of cantilever response allows two vector components, in-plane and out-of-plane, to be simultaneously measured. (b) Surface topography (vertical scale $20 \mathrm{~nm}$ ) and (c) elasticity map of a $400 \times 400 \mathrm{~nm}^{2}$ region on the enamel surface (vertical scale is $6 \%$ of the average signal). Vertical (d) and lateral (e) PFM images of the same region as (a, b) with a modulation bias of $10 V_{\text {pp }}$ applied to the tip. The vertical scale for (d) is -7.5 to $7.5 \mathrm{pm} / \mathrm{V}$ (the vertical scale for (e) is not calibrated). (f) Vector PFM map of local electromechanical response (maximum is $7.5 \mathrm{pm} / \mathrm{V}$ ). Color indicates the orientation of the electromechanical response vector, while the intensity provides the magnitude (color wheel diagram). (g, h) Semiquantitative map of local molecular orientation.

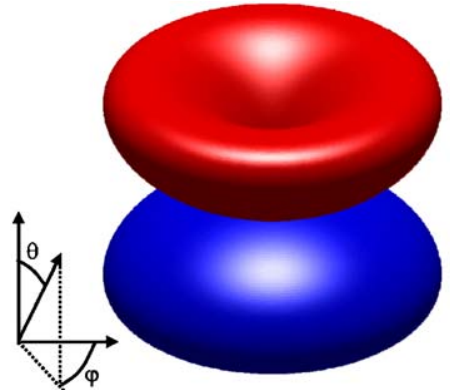

(a)

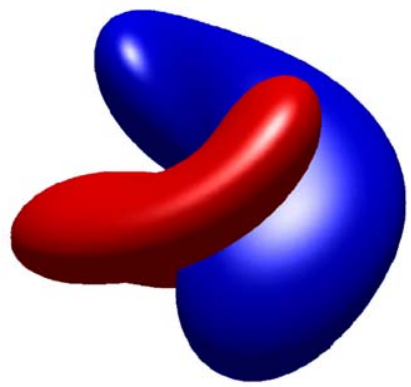

(b)
Fig. 4. Orientation dependence of the absolute value of (a) vertical and (b) lateral PFM signals for collagen (blue - negative, red - positive). Note that while the exact shape of the response surfaces will depend on the precise values of the elements of the piezoelectric constant tensor, the nodes are determined by the crystal symmetry only.

the nodes are dependent only on the crystal symmetry and will be universal for most biological systems having shear piezoelectricity.

For rotationally invariant molecules, the combination of VPFM and LPFM data is sufficient for the reconstruction of molecular orientation. We use the $d_{i j}^{0}$ values for collagen reported by Gunjian [30]

$$
\left(\begin{array}{cccccc}
0 & 0 & 0 & -26.6 & 14.0 & 0 \\
0 & 0 & 0 & 14.0 & 26.6 & 0 \\
0.666 & 0.666 & 0.866 & 0 & 0 & 0
\end{array}\right),
$$

and assume $d_{14}^{0}, d_{15}^{0} \gg d_{33}^{0}, d_{13}^{0} \approx 0$ (in $\left.\mathrm{pm} / \mathrm{V}\right)$. In this case, signals measured in the PFM experiment are proportional to $d_{33}=\cos \theta(7-7 \cos 2 \theta)$ and $d_{34}=(26.6 \cos \theta \sin$ $\psi-14 \cos \psi \cos 2 \theta) \sin \theta$, providing two linearly independent equations to determine angles, $(\theta, \psi)$. Given the large degree of uncertainty in published values for $d_{i j}^{0}$ and lack of absolute calibration, VPFM and LPFM signals were normalized by $d_{33}^{\max }=\max \left[d_{33}(\theta, \psi)\right], d_{34}^{\max }=\max$ $\left[d_{34}(\theta, \psi)\right]$, where $(\theta, \psi) \in(0,2 \pi)$, providing a semiquantitative calibration to relate measured PFM signal to local electromechanical response. The numerical solution of Eqs. $(3,4)$ yields two real branches. Given that the solution should be continuous, the first branch is selected as an orientation map in the experiment and thus obtained orientation maps are shown in Fig. 3g, h.

It should be noted that the absence of reliable piezoelectric data and the partial knowledge of the electromechanical response vector (only 2 of 3 orthogonal 
components are measured) limits the orientation imaging in this case to semiquantitative at best. However, we expect that the potential for orientation imaging of biological systems on the sub- $10 \mathrm{~nm}$ level will stimulate further development of this approach (e.g. using complete 3D PFM data), and on current theoretical modeling on the calculation of electromechanical coupling coefficients for complex biomolecules, potentially opening pathways to molecular identification.

\subsection{Piezoelectric imaging of a butterfly wing}

While teeth are robust, the majority of biological objects are either too fragile or too small to be studied by macroscopic methods. We demonstrate SPM imaging of local structure, elastic and electromechanical properties of a butterfly wing, on length scales from 50 to $10 \mathrm{~nm}$. Shown in Fig. 5a is the butterfly Vanessa virginiensis (American Lady). The wing was glued using silver paint to a silicon wafer, which was then mounted on the piezoelectric oscillator.

A butterfly wing is covered with thousands of pigmented chitin scales as shown in a digital optical microphotograph in Fig. 5b. The top surfaces of the scales exhibit intricate microstructure consisting of ridges extending longitudinally and connected by a series of crossribs (Fig. 5c). Figs. 5d and e show simultaneously acquired SPM surface topography and AFAM images of the wing. Note the difference between the effective resolution of the two images-while no features smaller than $100 \mathrm{~nm}$ can be distinguished in the topographic image, the AFAM image shows details with sub-10 nm resolution. Significant varia- tions in elastic contrast are seen within an individual ridge, suggesting an internal structural inhomogeneity. Due to significant variations in local topography, the contrast can be attributed both to local property variations within the wing and topographic features. However, whether the contrast is due to topographic or property variations, the effective resolution of AFAM is expected to be enhanced compared to the topographic imaging, since topographic variations on the length scales larger than contact area contribute to the AFAM signal only weakly.

The unique mechanical properties of butterfly wings originate from the scale structure, a biological composite of piezoelectric chitin rods [9] embedded in a protein matrix. A vector PFM map of a $5 \times 5 \mu \mathrm{m}^{2}$ region on the scale surface (Fig. 5f) reveals position-dependent piezoelectric properties: uniform response within the ridges and in the membrane and varying between these structural elements. The effective piezoelectric constant was estimated as $\sim 1 \mathrm{pm} / \mathrm{V}$. Colors in the vector PFM image are assigned to the directions of the surface electromechanical response vector, which in turn are related to the dominant orientation of chitin fiber.

Note that the symmetry of the piezoelectric constant tensor, which is closely related to the crystal structure of the material, ultimately determines the shape of the piezoresponse surface in Fig. 4. In particular, for a material with the symmetry of collagen (tetragonal class 4) the response is zero perpendicular to the molecular axis. If chitin is also a shear piezoelectric, the non-zero vertical response observed for the butterfly wing indicates that the molecules are not simply oriented linearly along the surface, rather, a more complex organization in which
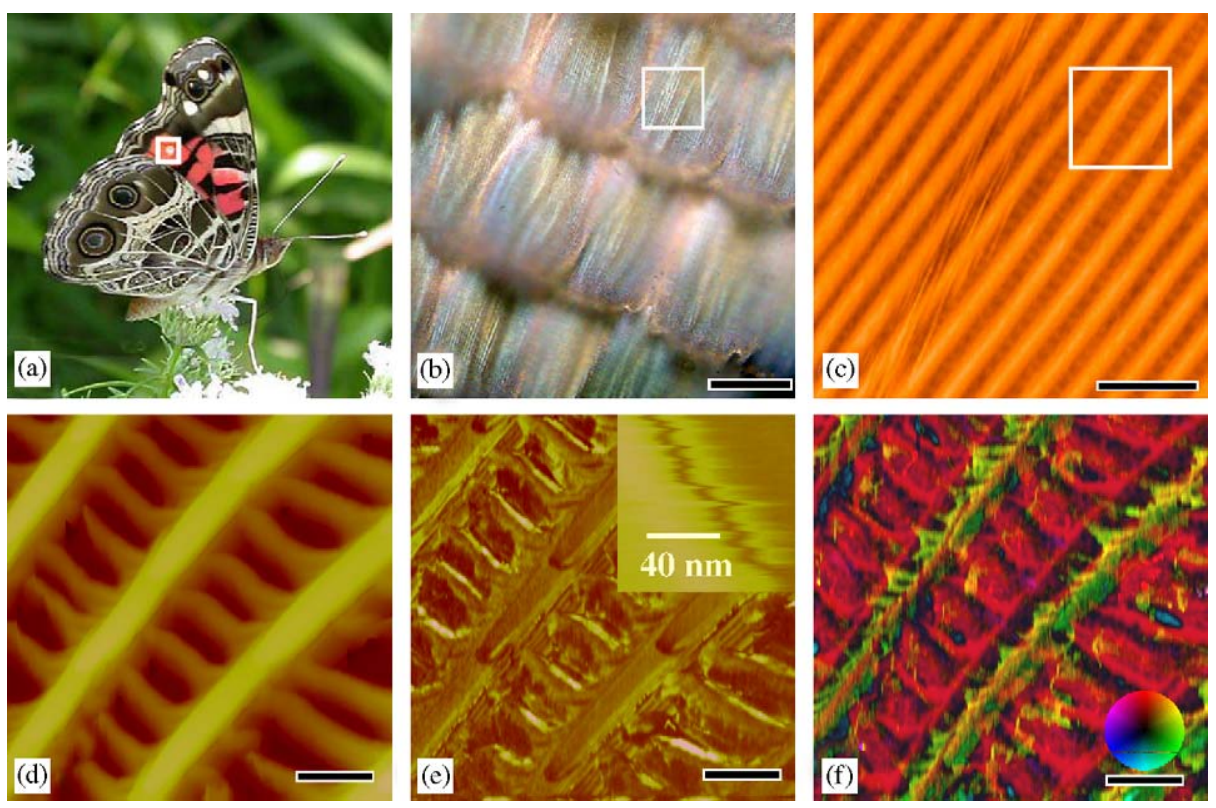

Fig. 5. (a) Optical photograph of Vanessa virginiensis (courtesy of Jeffrey Pippen, Duke University) and (b) optical micrograph of the wing scales. (c, d) AFM surface topography of the wing (vertical scale is $1 \mu \mathrm{m}$ ). (e) Elasticity image (vertical scale is $18 \%$ of the average signal) of the wing obtained simultaneously with (d). Inset shows high-resolution segment, illustrating sub-10 nm spatial resolution in the acoustic regime. (f) Vector PFM image of the same location on the wing (maximum is $\sim 1 \mathrm{pm} / \mathrm{V}$ ). Scale is 50 (b), 5 (c), and $1 \mu \mathrm{m}(\mathrm{d}-\mathrm{f}$ ). 
individual molecules are oriented at an angle to the surface, is more likely. Also, note that the effective orientation of the response vector in the cross-ribs and ridges differs by approximately $90^{\circ}$, suggesting a misorientation between the fibers, in agreement with observed structure in topographic image.

Thus, despite being semiquantitative and the lack of piezoelectric data for biological systems (which can be obtained from first principle calculations, as has been demonstrated for materials such as poly(vinylidene fluoride) [31]) PFM provides a measure of local orientation and disorder in a material with sub- $10 \mathrm{~nm}$ resolution.

\section{Conclusions}

To summarize, the explicit relationship between piezoelectric and mechanical properties and molecular orientation and biological functionality is key to understanding biological systems. The SPM approach for simultaneous imaging of electromechanical and elastic properties provides a new tool to study these properties on the hierarchy of length scales from tens of microns to $10 \mathrm{~nm}$, i.e. on the length scale of the individual building blocks of the structural properties. The ability to obtain information on the molecular orientation of electromechanically active proteins such as collagen and chitin based on $2 \mathrm{D}$ vector PFM is demonstrated, and the applicability of rigorous molecular orientation determination is proposed. Molecular orientation in real space will be enabled by improved modeling of the piezoelectric tensor for biosystems and the measurement of VPFM and two orthogonal directions of LPFM of the same area are required. The SPM method will potentially play a crucial role in improving the understanding of the structure-property-functionality relationship and molecular orientation imaging in biosystems.

\section{Acknowledgments}

Research performed in part as a Eugene P. Wigner Fellow and staff member at the Oak Ridge National Laboratory, managed by UT-Battelle, LLC, for the US Department of Energy under Contract DE-AC0500OR22725 (SVK). Support from ORNL SEED funding is acknowledged (SVK and TGT). AG acknowledges financial support of the National Science Foundation (Grant no. DMR02-35632).

\section{References}

[1] L. Galvani. De Viribus Electricitatis in Motu Musculari Commentarius, Antichi Commentari, Bologna (1791). Commentary on the effect of electricity on muscular motion, English translation by R.M. Green (Cambridge, MA, 1953).

[2] E. Fukada, I. Yasuda, J. Phys. Soc. Japan 12 (1957) 1158.

[3] L. Yasuda, J. Jpn. Orthop. Surg. Soc. 28 (1957) 267.

[4] S.B. Lang, Nature 5063 (1966) 704.

[5] J.C. Anderson, C. Eriksson, Nature 227 (1970) 491.

[6] A.A. Marino, B.D. Gross, Arch. Oral Biol. 34 (7) (1989) 507.

[7] E. Fukada, J. Phys. Japan 10 (1955) 149.

[8] V.A. Bazhenov, Piezoelectric Properties of Wood, Consultants Bureau, New York, 1961.

[9] E. Fukada, Biorheology 32 (6) (1995) 593.

[10] C.A.L. Bassett, Calc. Tissue Res. 1 (1968) 252.

[11] A.A. Marino, R.O. Becker, Nature 228 (1970) 473.

[12] B. Ji, H. Gao, J. Mech. Phys. Solids 52 (2004) 1963.

[13] S. Weiner, H.D. Wagner, Annu. Rev. Mater. Res. 28 (1998) 271.

[14] P. Fratzl, S. Schreiber, K. Klaushofer, Connect. Tissue Res. 35 (1-4) (1996) 9.

[15] W.J. Landis, Bone 16 (1995) 533.

[16] G. Willems, J.P. Celis, P. Lambrecht, M. Braem, G. Vangherle, J. Biomed. Mater. Res. 27 (1993) 747.

[17] C. Halperin, S. Mutchnik, A. Agronin, M. Molotskii, P. Urenski, M. Salai, G. Rosenman, Nano Letters 4 (2004) 1253.

[18] S.V. Kalinin, B.J. Rodriguez, S. Jesse, T. Thundat, A. Gruverman, Appl. Phys. Lett. 87 (2005) 053901.

[19] J. Shin, B.J. Rodriguez, A.P. Baddorf, T. Thundat, E. Karapetian, M. Kachanov, A. Gruverman, S.V. Kalinin, J. Vac. Sci. Technol. B 23 (2005) 2102.

[20] B.J. Rodriguez, S.V. Kalinin, J. Shin, S. Jesse, V. Grichko, T. Thundat, A. Gruverman, 2005, submitted.

[21] A. Gruverman, in: H.S. Nalwa (Ed.), Encyclopedia of Nanoscience and Nanotechnology, vol. 3, American Scientific Publishers, Los Angeles, 2004, pp. 359-375.

[22] S.V. Kalinin, E. Karapetian, M. Kachanov, Phys. Rev. B 70 (2004) 184101.

[23] S.V. Kalinin, B.J. Rodriguez, S. Jesse, J. Shin, A. P. Baddorf, P. Gupta, H. Jain, D. B. Williams, A. Gruverman, Microscopy and Microanalysis, 2005, accepted.

[24] A. Kholkin, I.K. Bdikin, V.V. Shvartsman, S.-H. Kim, J.M. Herrero, A.L. Kholkin, in: S. Hoffmann-Eifert, H. Funakubo, V. Joshi, A.I. Kingon, I.P. Koutsaroff (Eds.), Ferroelectric Thin Films XII, Material Research Society Symposium Proceedings, vol. 784, Warrendale, PA, 2004, p. C11.3.

[25] U. Rabe, M. Kopycinska, S. Hiserkorn, J. Munoz-Saldana, G.A. Schneider, W. Arnold, J. Phys. D 35 (2002) 2621.

[26] SD 0.394-0.000-0.040-502, Piezo Kinetics, Bellefonte, PA 16823, www.piezo-kinetics.com

[27] Mathematica 5.0, Wolfram Research.

[28] J.W. Smith, Nature 219 (1968) 157.

[29] R.E. Newnham, Properties of Materials: Anisotropy, Symmetry, Structure, Oxford University Press, Oxford, 2005.

[30] A.A. Gunjian, H.L. Chen, IEEE Trans. Biomed. Eng. 21 (3) (1974) 177-182.

[31] S.M. Nakhmanson, M.B. Nardelli, J. Bernholc, Phys. Rev. Lett. 92 (2004) 115504-115507. 